multiple diagnoses and the evolution of different potential pain generators over time can make obtaining the correct etiology, such as spinal stenosis, primary hip pathology, or sacral fractures, a significant challenge.

Conclusions: When evaluating the chief complaint of low back pain and extremity pain in the elderly stenotic patient, one must explore all diagnostic entities and continuously remember the vast differential diagnosis. If treatment is unsuccessful, reassessment and consideration of alternative diagnoses is essential.

\section{Poster 294}

\section{The Use of Baclofen in the Treatment of Thalamic Pain Syndrome: A Case Report. Jennifer Kendall, DO (University of Michigan, Ann Arbor, MI); Joseph E. Hornyak, MD, PhD.}

Disclosures: J. Kendall, None.

Patients or Programs: A 7-year-old boy with thalamic pain syndrome from an inoperable brain tumor.

Program Description: The patient was diagnosed with a teratoid/rhabdoid brain tumor involving the right superior brainstem and thalamus. He developed severe pain in his left hand, which progressed to involve his left forearm. He had been treated with simultaneous long-acting oxycodone, nortriptyline, and gabapentin, with little relief. On physical examination he had a mildly increased tone in his left upper extremity as well as hyperalgesia and allodynia. Oxycodone, nortriptyline, and gabapentin were discontinued, and he was started on oral baclofen $10 \mathrm{mg}$ at bed time, primarily for pain control. The patient had significant improvement in his pain, with even occasional total resolution of pain. Pain resumed after baclofen was stopped during a hospitalization. Resumption of baclofen again markedly improved pain control.

Setting: University outpatient and inpatient settings.

Results: Significant decrease in pain while on oral baclofen, worsening when baclofen discontinued.

Discussion: Central pain syndromes are neurologic conditions caused by damage to or dysfunction of the central nervous system, in this case thalamic in origin. Thalamic pain syndrome is commonly treated with tricyclic antidepressants or anticonvulsants. Baclofen is a $\gamma$-aminobutyric acid B (GABA-B) agonist that is commonly used to treat spasticity associated with upper motor neuron syndrome. GABA plays numerous roles in the central nervous system and is thought to play an important role in anti-nociception. Anti-nociceptive effects of baclofen have been demonstrated, with most evidence surrounding the treatment of trigeminal neuralgia and pain associated with spinal lesions. This case developed into an inadvertent A-B-A trial, with marked pain relief from baclofen.

Conclusions: Baclofen may have a role in decreasing pain in patients with thalamic pain syndrome.

\section{PEDIATRICS}

\section{Poster 296}

Blood Pressure and Obesity in Ambulatory Children and Adolescents With Cerebral

Palsy.

Elizabeth A. Moberg-Wolff, MD (Children's Hospital of Wisconsin); Darcy Fehlings; Deborah J. Gaebler-Spira, MD; Edward A. Hurvitz, MD; Linda E. Krach, MD; Maxine M. Kuroda, PhD, MPH; Kat Kolaski, MD; Lisa Thornton, MD.

Disclosures: E.Moberg-Wolff, None.

Objective: Recent studies have documented an association between childhood obesity and hypertension with premature adult mortality. This study reports frequency of high blood pressure (BP) and obesity in ambulatory children and adolescents with cerebral palsy (CP), and explores their relationship to each other and to severity of motor function. Design: Cross-sectional.

Setting: 5 clinic sites in the United States and Canada. Participants: $N=87$ (43M, 24F), a subset of 112 participants used in a feasibility study for the multicenter Cerebral Palsy Outcomes Project (CPOP). Participants were aged 6-17 years and had a diagnosis of $\mathrm{CP}$, with motor function rated level I-III with the Gross Motor Functional Classification System (GMFCS).

Main Outcome Measures: Measures included weight, height, and BP. Gender and age-specific BMI percentiles were calculated and categorized as underweight, healthy weight, overweight, or obese by using parameters from the CDC. BP percentiles were determined by using $\mathrm{BP}$ readings and height percentiles from the CDC. Hypertension was defined by criteria of the National High Blood Pressure Education Program.

Results: Gender (M:F=1.5:1) and race/ethnicity were similar to published reports on CP and with U.S. Census Bureau statistics. Participants in the 3 GMFCS levels did not differ by age (mean, $10.8 \pm 3.6 \mathrm{y}$ ), gender, race/ethnicity, socioeconomic status, or BMI percentile. Approximately $30 \%$ of participants were overweight or obese (>85th BMI percentile) based on gender and age norms for typically developing children. Abnormally high systolic (S) BP was found in approximately $23 \%$ of the participants and in 20\% for diastolic (D) BP. Neither SBP nor DBP were associated with gender, age, or BMI percentile, but both had a positive linear association with GMFCS.

Conclusions: An unexpectedly high frequency of 2 major cardiovascular risk factors in ambulatory children with $\mathrm{CP}$ was found. Notably, these BMI data might underestimate risk because of low lean body mass in CP. Further study on a larger population is needed. Future studies will be strengthened by designs that ensure accurate, reproducible measurements, and compliance with measurement protocols in 